\title{
Process-dependent band structure changes of transition-metal (Ti,Zr,Hf) oxides on Si (100)
}

\author{
C. C. Fulton, G. Lucovsky, and R. J. Nemanich ${ }^{\mathrm{a})}$ \\ Department of Materials Science and Engineering and Department of Physics, \\ North Carolina State University, Raleigh, North Carolina 27695-8202
}

(Received 16 July 2003; accepted 15 November 2003)

\begin{abstract}
In this study, we have deposited $\mathrm{Ti}, \mathrm{Zr}$, and $\mathrm{Hf}$ oxides on ultrathin $(\sim 0.5 \mathrm{~nm}) \mathrm{SiO}_{2}$ buffer layers and have identified metastable states which give rise to large changes in their band alignments with respect to the $\mathrm{Si}$ substrate. This results in a potential across the interfacial $\mathrm{SiO}_{2}$ layer, significant band bending, and large shifts of the high- $k$ valence band. The magnitude of the shift differs for the three materials and is dependant on both the $\mathrm{SiO}_{2}$ buffer layer thickness and annealing temperature. We propose a model where excess oxygen accumulates near the high- $k-\mathrm{SiO}_{2}$ interface providing electronic states, which are available to electrons that tunnel from the substrate. () 2004 American Institute of Physics. [DOI: 10.1063/1.1639944]
\end{abstract}

The task of identifying a suitable replacement dielectric for high-performance, low-power devices is complex with many difficulties. Transition-metal (Tm) oxides have received much attention for their large dielectric constants and relatively large band gaps. Zirconium and hafnium oxides, in particular, have been considered. Titanium oxides have also received attention but, due to their smaller band gap, they have generally not received the same consideration as potential replacement dielectrics for field effect transistors (FETs).

The primary goal in choosing a replacement for $\mathrm{SiO}_{2}$ is to reduce the tunneling current through the gate oxide of a FET while maintaining the channel charge density. There are two main parameters that effect tunneling current: (1) the physical thickness of the dielectric and (2) the barrier height or band offset with respect to Si. Choosing a high- $k$ material with a large dielectric constant can solve the tunneling problem by increasing the dielectric thickness, but these materials often have barrier heights smaller than the traditional $\mathrm{SiO}_{2}$ on $\mathrm{Si}$, sometimes very much smaller. For instance, $\mathrm{TiO}_{2}$ has essentially no barrier with respect to the Si conduction band, and electrons would be free to move from channel to gate, through the oxide. ${ }^{1}$

Understanding barrier heights and how they can be altered by different process conditions is a critical part of choosing a replacement dielectric material. In this letter, we report an effect that can alter barrier heights by as much as $2.0 \mathrm{eV}$. We determine the circumstances where the effect occurs and propose a model to explain these large shifts in band structure.

All experiments employed $n$-type $25 \mathrm{~mm}$ diameter $\mathrm{Si}(100)$ wafers with phosphorous doping of $\sim 4$ $\times 10^{18} \mathrm{~cm}^{-3}$. The doping density is calculated from the lowest value in the resistivity range quoted from the manufacturer. All processing and characterization occurred in UHV conditions. The $\mathrm{Si}$ wafers were prepared with a $\mathrm{SiO}_{2}$ buffer layer by either remote plasma oxidation or remote plasma enhanced chemical vapor deposition. In this study, $\mathrm{Tm}$ oxides were formed by electron beam evaporation of a

${ }^{a)}$ Electronic mail: robert_nemanich@ncsu.edu metal ( $\mathrm{Ti}, \mathrm{Zr}$, or $\mathrm{Hf}$ ) onto the $\mathrm{SiO}_{2}$ buffer layer followed by remote plasma oxidation. The electronic states were then characterized with x-ray and ultraviolet photoelectron spectroscopy (XPS,UPS). A more detailed description of this approach has been presented previously. ${ }^{1}$

In characterizing the band offsets of the Tm as-deposited oxides on a $\mathrm{SiO}_{2}$ buffer, we observed a large shift in the valence band turn-on and all of the oxide core levels when the films were annealed to $500^{\circ} \mathrm{C}$. This effect was found to occur in $\mathrm{Ti}, \mathrm{Zr}$, and $\mathrm{Hf}$ oxides, with $\mathrm{ZrO}_{2}$ demonstrating the largest shift. Figure 1 shows UPS spectra for all three oxides in their as-grown state (solid lines) and in their annealed state (dotted). With annealing, the $\mathrm{ZrO}_{2}$ valence-band maximum (VBM) was shown to shift up to $\sim 2.0 \mathrm{eV}$, while the $\mathrm{HfO}_{2}$ and $\mathrm{TiO}_{2}$ shifted by $\sim 0.8 \mathrm{eV}$, depending on process conditions. Accounting for changes in band bending, we deduce the Si-high- $k$ valence band offset (VBO) to be 1.65, 0.95, and $2.05 \mathrm{eV}$ for the as-grown $\mathrm{Ti}, \mathrm{Zr}$, and $\mathrm{Hf}$ oxides, respectively, and after annealing, the VBO increase to 2.55, 3.0, and $2.75 \mathrm{eV}$ for the respective $\mathrm{Ti}, \mathrm{Zr}$, and $\mathrm{Hf}$ oxides.

We previously reported the $\mathrm{VBO}$ of $2.2 \mathrm{eV}$ for $\mathrm{TiO}_{2}$ which falls between the shifted and annealed values we observe in this study, ${ }^{1}$ and Campbell et al. found a VBO of 1.0

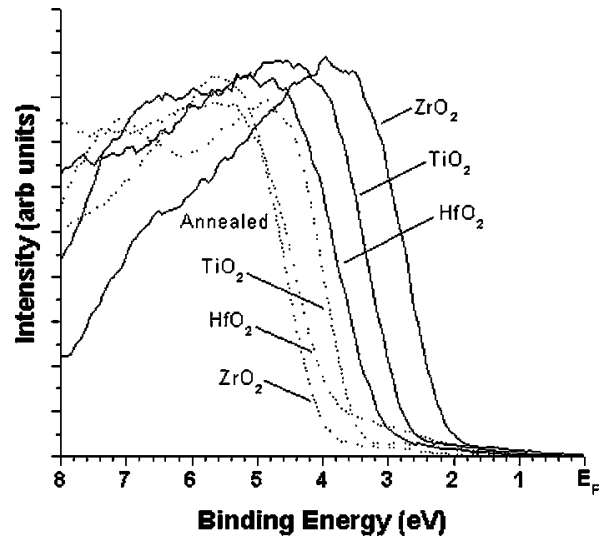

FIG. 1. UPS valence band spectra for $\mathrm{Ti}, \mathrm{Zr}$, and $\mathrm{Hf}$ oxides with a $\mathrm{SiO}_{2}$ buffer layer on $\mathrm{Si}$. The solid lines indicate the as-grown state and the dotted lines indicate the annealed state. 


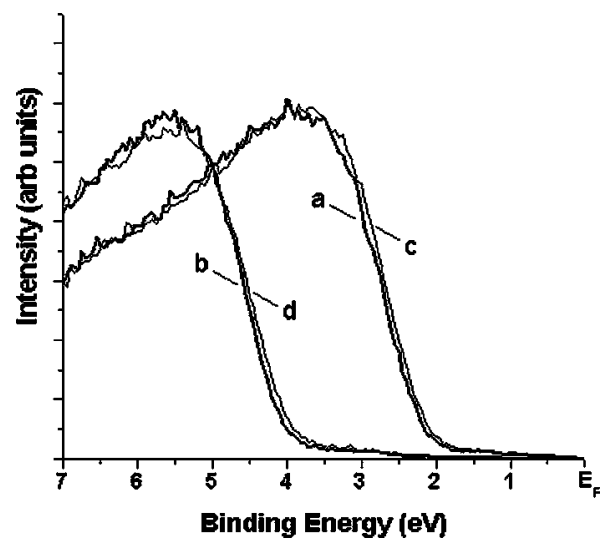

FIG. 2. UPS spectra of a $\mathrm{ZrO}_{2}$ film with a $\mathrm{SiO}_{2}$ buffer layer on $\mathrm{Si}$. The spectra were obtained (a) as grown, (b) $500{ }^{\circ} \mathrm{C}$ anneal, (c) reoxidized, and (d) second $500^{\circ} \mathrm{C}$ anneal.

$\mathrm{eV}$ which is near the value of the as-deposited film. ${ }^{2,3}$ In a study of $\mathrm{ZrO}_{2}$ on Si (100), Miyazaki et al., utilizing XPS, found a valence band offset of $3.2 \mathrm{eV},{ }^{4-6}$ which is in reasonable agreement with our measurements of the annealed film. Predictions made by Robertson using the charge neutrality model obtain a VBO of 2.0 and $3.3 \mathrm{eV}$ for $\mathrm{TiO}_{2}$ and $\mathrm{ZrO}_{2}$, respectively. ${ }^{7}$ For $\mathrm{HfO}_{2}$ Robertson has noted that the charge neutrality model predicts a VBO of $3.4 \mathrm{eV}$. Sayan et al. have used photoemission to measure the $\mathrm{VBO}$ of $\mathrm{HfO}_{2}$ with respect to $\mathrm{Si}$ and found values between 3.03 and $3.63 \mathrm{eV},{ }^{8}$ which is in relative agreement with the predictions and our measurements. However, Yu et al. have found that their asgrown $\mathrm{HfO}_{2}$ films showed a VBO of $2.22 \mathrm{eV},{ }^{9}$ which is similar to our observations of the as-grown state.

We have explored the origin of this effect through a process of re-exposing the annealed films to the remote plasma oxidation. As shown in Fig. 2 the VBM of the as-grown and reoxidized spectra are essentially identical, as are the two annealed spectra. The core level spectra of the Tm oxide shifted in a similarly consistent way suggesting that the shifts are of electronic, not chemical, origin. Similar films were annealed and characterized in a stepwise fashion at temperatures of $200,300,400$, and $500{ }^{\circ} \mathrm{C}$. The photoelectron spectra shifted gradually at low temperatures and shifted to a greater extent at higher temperatures.

The core levels of the Tm and the Tm oxide-related $\mathrm{O} 1 \mathrm{~s}$ core level spectra showed similar shifts to the valence band. An interesting aspect was evident on examination of the $\mathrm{SiO}_{2}$ related $\mathrm{O} 1 s$ and $\mathrm{Si} 2 p$ core levels. The core level spectra associated with the buffer layer shifted to a lesser degree than the Tm oxide core levels and the valence band spectra. Figure 3 shows the $\mathrm{O} 1 \mathrm{~s}$ spectra for an as-grown and annealed $\mathrm{ZrO}_{2}$ film. The strongest spectral feature (at lower binding energy) is associated with the $\mathrm{ZrO}_{2}$ which, upon annealing, shifted by a magnitude equal to that of the valence band maxima. The weaker feature, at slightly higher binding energy, is associated with the $\mathrm{SiO}_{2}$ buffer layer, which shifted less than the $\mathrm{ZrO}_{2}$ component.

We also explored how variations in the thickness of the $\mathrm{SiO}_{2}$ buffer layer affect the changes in band structure. Buffer layers were prepared with thicknesses of $0.5,2.0$, and $3.5 \mathrm{~nm}$ followed by the deposition of $1.2 \mathrm{~nm}$ of $\mathrm{HfO}_{2}$. Valence band and core level spectra were recorded, and the shift between Downloaded 22 Feb 2008 to 152.1.190.114. Redistribution subject to

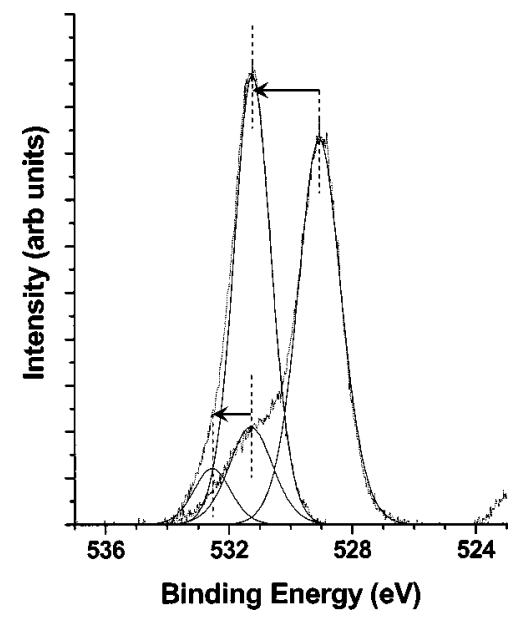

FIG. 3. XPS O $1 s$ core level of an as-grown and annealed $\mathrm{ZrO}_{2}$ film with a $\mathrm{SiO}_{2}$ buffer on $\mathrm{Si}$. The larger peaks are associated with the $\mathrm{ZrO}_{2}$ layer and the smaller peaks with the buried $\mathrm{SiO}_{2}$ buffer layer. The shifts on annealing (arrows) are larger for the $\mathrm{ZrO}_{2}$ than the $\mathrm{SiO}_{2}$ buffer layer.

the as-grown and annealed states was measured. For the 0.5 $\mathrm{nm}$ buffer layer, a VB shift of $1.50 \mathrm{eV}$ was measured. The shifts in the core levels and valence band decrease as the buffer layer thickness is increased. Increasing the thickness of the buffer to $2.0 \mathrm{~nm}$ resulted in a $1.35 \mathrm{eV}$ shift. A further increase in buffer layer thickness to $3.5 \mathrm{~nm}$ resulted in a VB shift of $0.20 \mathrm{eV}$.

We suggest that the exposure to a remote oxygen plasma (and a high concentration of atomic oxygen) introduces excess oxygen into the high- $k$ material and that this oxygen is in a metastable state where annealing can apparently drive out the excess.

On first examination one might suggest that an interface dipole has formed at the Si-buffer layer interface. However, such a dipole would shift the entire band structure equally, meaning that the $\mathrm{SiO}_{2}$ and $\mathrm{ZrO}_{2}$ electronic states would shift by the same magnitude and direction. This was not the observed case; instead the spectra associated with the $\mathrm{SiO}_{2}$ buffer layer and Tm oxide shifted in the same direction but by a different magnitude, suggesting that there is a potential drop across the buffer layer. We propose that excess negative charge is present near the high- $k-\mathrm{SiO}_{2}$ interface and that there is consequently a potential across the buffer oxide, between the substrate and the charge layer in the high- $k$ material. Figure 4(a) displays a schematic of the bands of the as-grown film with excess charge at the high- $k$ interface and a potential across the buffer layer, and Fig. 4(b) shows the annealed state with flat bands, no excess charge and no potential over the buffer layer.

Fissel et al. have noted similar core level shifts for $\mathrm{PrO}_{2}$ on $\mathrm{Si}$ and have suggested a model, which assumes no interfacial layer of $\mathrm{SiO}_{2}$ and an epitaxial oxide on the $\mathrm{Si}$ substrate. ${ }^{10}$ In our case, with the purposeful formation of an interfacial oxide, it is unlikely that our Tm oxides are epitaxial nor is our growth method conducive to forming single crystal oxides. As such, we must explore other possibilities in explaining our observations.

Our results indicate that the total electronic shift falls off as a function of increasing buffer layer thickness; approaching zero as the buffer layer grows past $3.5 \mathrm{~nm}$. If the charge were constant near the interface then the potential difference AIP license or copyright; see http://apl.aip.org/apl/copyright.jsp 


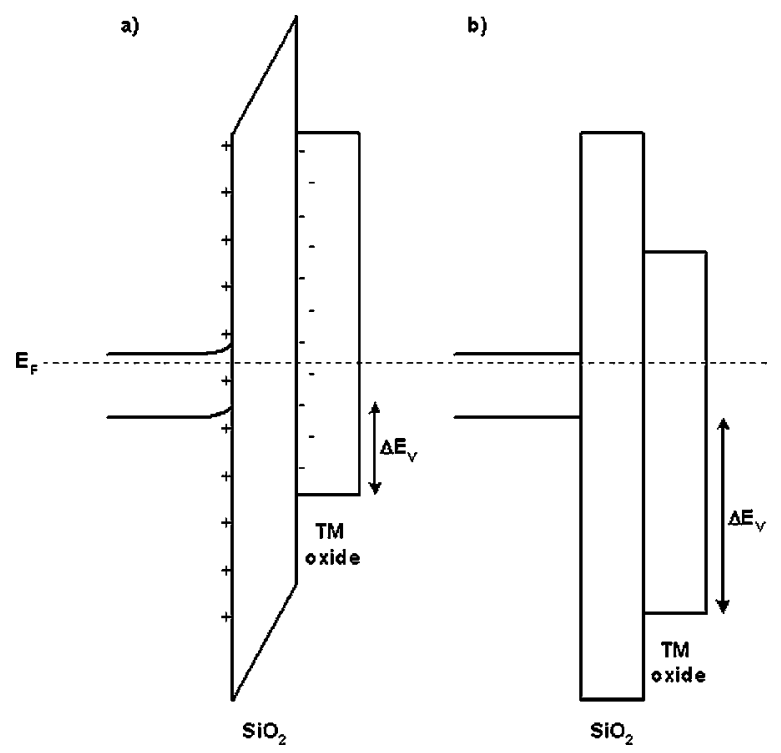

FIG. 4. Schematic diagram of the as-grown (a) and annealed (b) state: (a) excess oxygen near the $\mathrm{Tm}$ oxide $-\mathrm{SiO}_{2}$ interface attracts electrons from the substrate, resulting in a potential across the $\mathrm{SiO}_{2}$ buffer layer; (b) the oxygen diffuses to vacuum, there are no interface electronic states and the bands become flat. The valence band offset $\left(\Delta E_{V}\right)$ can change by up to $2.0 \mathrm{eV}$ with annealing.

across the buffer layer would increase with buffer layer thickness. This is not the observed case, and, in fact, the decreasing potential suggests that the amount of charge decreases with increasing buffer layer thickness. We propose that the charge layer is controlled by a tunneling process from the substrate.

The tunneling probability of an electron through $\mathrm{SiO}_{2}$ falls off exponentially, with the probability becoming small after 3-4 nm thickness. We suggest that electrons tunnel from the substrate, though the buffer layer, to satisfy the electronic properties of the excess oxygen in the high- $k$ material. The Tm oxides, $\mathrm{ZrO}_{2}$ in particular, are known to be ionic conductors where the majority ion (oxygen) may move with relative ease. ${ }^{11}$ We suggest the following as a possible scenario: oxygen diffuses into the high- $k$ dielectric and electrons tunnel from the substrate, through the $\mathrm{SiO}_{2}$ buffer layer to satisfy the electronic states of the excess oxygen. This results in a potential across the buffer oxide and a shift in the band structure of the high- $k$ material. When the source of oxygen is removed, the oxygen diffuses from the interface and escapes to vacuum. This diffusion of oxygen can be accelerated with annealing.
To estimate the charge needed to produce the measured shifts, we treat the buffer layer as a simple dielectric filled capacitor with thickness of $d=2.0 \mathrm{~nm}$, a dielectric constant of $k=3.9$ for $\mathrm{SiO}_{2}$ and a voltage of $V=1.0 \mathrm{~V}$. From this we determine a charge density of $\sim 1 \times 10^{13} \mathrm{~cm}^{-2}$. We can likewise estimate the positive charge in the $\mathrm{Si}$ from the change in band bending at the $\mathrm{Si}$ substrate. We use $N_{s}$ $=\sqrt{2 k \epsilon_{0} N_{D} V / q}$ to calculate the charge density, where $N_{s}$ is the surface charge density, $N_{D}=\sim 4 \times 10^{18} \mathrm{~cm}^{-3}$ is the bulk doping density of the $\mathrm{Si}, q$ is the charge of an electron, $\epsilon_{0}$ is the permittivity of free space, $k=12$ is the dielectric constant of $\mathrm{Si}$, and $V=0.3 \mathrm{~V}$ is the change in band bending. This gives a charge density of $N_{s}=4 \times 10^{12} \mathrm{~cm}^{-2}$. The charge may not be completely localized at the high- $k-\mathrm{SiO}_{2}$ interface or other conditions may exist to explain the discrepancy in the two calculations.

This study has demonstrated that the electronic structure of the $\mathrm{Tm}$ oxide- $\mathrm{SiO}_{2}$ interfaces can be significantly influenced by process conditions. The excess oxygen state of this interface is metastable and would certainly lead to time dependent device characteristics. It remains to be seen whether processing approaches could be employed to improve the device characteristics. Correlated interface and device studies are suggested to quantify the effect of the charge layers and internal fields.

This work is supported through the Semiconductor Research Corporation, the Office of Naval Research, the Air Force Office of Scientific Research, and the Microelectronics Advanced Research Corporation.

${ }^{1}$ C. C. Fulton, G. Lucovsky, and R. J. Nemanich, J. Vac. Sci. Technol. B 20, 1726 (2002)

${ }^{2}$ S. A. Campbell, D. C. Gilmer, X. Wang, M. T. Hsich, H. S. Kim, W. L. Gladfelter, and J. H. Yan, IEEE Trans. Electron Devices 44, 104 (1997).

${ }^{3}$ S. A. Campbell, H. S. Kim, D. C. Gilmer, B. He, T. Ma, and W. L. Gladfelter, IBM J. Res. Dev. 43, 383 (1999).

${ }^{4}$ S. Miyazaki, J. Vac. Sci. Technol. B 19, 2212 (2001).

${ }^{5}$ S. Miyazaki, M. Narasaki, M. Ogasawaga, and M. Hirose, Solid-State Electron. 46, 1679 (2002).

${ }^{6}$ S. Miyazaki, M. Narasaki, M. Ogasawaga, and M. Hirose, Microelectron. Eng. 59, 373 (2001).

${ }^{7}$ J. Robertson, J. Vac. Sci. Technol. B 18, 1785 (2000).

${ }^{8}$ S. Sayan, E. Garfunkel, and S. Suzer, Appl. Phys. Lett. 80, 2135 (2002).

${ }^{9}$ H. Y. Yu, M. F. Li, B. J. Cho, C. C. Yeo, M. S. Joo, D.-L. Kwong, J. S. Pan, C. H. Ang, J. Z. Zheng, and S. Ramanathan, Appl. Phys. Lett. 81, 376 (2002).

${ }^{10}$ A. Fissel, J. Dabrowski, and H. J. Osten, J. Appl. Phys. 91, 8986 (2002).

${ }^{11}$ R. V. S. Stubican, G. S. Corman, J. R. Hellmann, and G. Senft, in Science and Technology of Zirconia II, Advances in Ceramics Vol. 12, edited by N. Claussen, M. Rühle, and A. H. Heuer (American Ceramic Society, Columbus, OH, 1984), pp. 96-106. 